\title{
Prevention of Postoperative Events Following Reversal With Sugammadex or Neostigmine (The P- PERSoN Trial) - Pilot Data Following Early Termination of a Prospective, Blinded, Randomised Trial.
}

Benjamin Luke Olesnicky ( $\square$ Benjamin.Olesnicky@health.nsw.gov.au )

Royal North Shore Hospital https://orcid.org/0000-0002-2650-7593

\section{Matthew Doane}

Royal North Shore Hospital Department of Anaesthesia

Clare Farrell

Westmead Hospital

\section{Greg Knoblanche}

Royal North Shore Hospital Department of Anaesthesia

\section{Anthony Delaney}

Royal North Shore Hospital

\section{Research}

Keywords: Sugammadex, Neostigmine, Postoperative Pulmonary Complications, Perioperative Medicine, Humans, Adult, Double-Blind Method

Posted Date: October 18th, 2021

DOl: https://doi.org/10.21203/rs.3.rs-960365/v1

License: (c) (i) This work is licensed under a Creative Commons Attribution 4.0 International License. Read Full License 


\section{Abstract}

Background: Residual paralysis following anaesthesia is common and can lead to postoperative morbidity. While sugammadex has been shown to be effective in minimising residual paralysis, uncertainty exists as to whether its use reduces any associated morbidity. We designed this trial to determine if the use of sugammadex for the reversal of intraoperative aminosteroid neuromuscular blockade results in improvements in postoperative pulmonary complications, complications in the recovery unit, postoperative nausea and vomiting, and patient satisfaction, when compared to reversal with neostigmine.

Methods: A prospective, double-blind, randomised controlled trial in adult patients admitted for surgical operations at two Australian hospitals between December 2018 and March 2019 was performed comparing the reversal of neuromuscular paralysis using sugammadex $2 \mathrm{mg} / \mathrm{kg}$ versus neostigmine $50 \mathrm{mcg} / \mathrm{kg}$. Statistical analysis of continuous data was performed using two tailed t-tests, with categorical and ordinal data was assessed by Chi-squared analysis.

Results: The trial was terminated due to a combination of resource constraints and the 2019 novel coronavirus disease (COVID-19) pandemic. Of 51 patients screened, 33 were eligible for participation and 30 subsequently recruited and randomised. All patients received the intended treatment allocated. Data for the primary outcome was obtained in all patients. There was no difference in the rates of postoperative pulmonary complications between the sugammadex and neostigmine groups $(0 \%(0 / 19)$ vs $9 \%(1 / 11)$ RR $5.0(95 \% \mathrm{Cl} 0.22-113) \mathrm{p}=0.37$. There was no difference in any of the secondary outcomes between the groups.

Conclusions. The P-PERSoN trial showed no difference in post operative pulmonary complications between sugammadex and neostigmine based reversal of aminosteroid neuromuscular block, but was underpowered to show any difference due to early trial termination. The randomisation and data collection was feasible. We support the need for an adequately resourced and funded randomised controlled trial to address this important clinical question.

Trial Registration: The P-PERSoN trial is registered at the Australian and New Zealand Trial Registry (www.anzctr.org.au) \# ACTRN12616000063415 and The U.S. National Library of Medicine (www.ClinicalTrials.gov) \#NCT02825576

\section{Key Messages Regarding Feasibility:}

- The ability to easily blind the administration of anaesthetic reversal agents and the ability to collect the selected data metrics across the recovery room, hospital, medical records and home was not known prior to this trial.

- Study randomsation processes, blinding and data collection as designed was easy to implement and feasible, however a subsequent large trial needs adequate infrastructure and funding to be completed. 
- Recruitment strategies and outcome measures could be used in a main trial. The use of an adapted quality of recovery score or a different score for 30 day follow up would be needed in a subsequent main trial.

\section{Background}

The practice of modern anaesthesia involves the use of muscle relaxants to provide muscle paralysis to enable surgical access and to allow effective mechanical ventilation. At the end of surgery, these muscle relaxants need to either have been cleared and no longer be active, or to be reversed, as significant residual relaxant activity will lead to residual paralysis. Residual paralysis is associated with increased patient morbidity. (1-5)

Sugammadex was introduced into clinical practice in Australia in 2009 and was specifically designed to reverse rocuronium by encapsulating it irreversibly, and blocking its activity at the neuromuscular end plate (6). Previous research had explored the effectiveness of sugammadex to prevent residual paralysis, consistently showing a reduction in the incidence of residual paralysis with sugammadex when compared with neostigmine $(5,7-9)$.

While sugammadex is shown to consistently reduce the incidence of residual paralysis, studies of the ability of sugammadex to prevent post-operative morbidity provide conflicting evidence. There has only been one published randomised prospective study looking at pulmonary complications occurring beyond discharge from the post-anaesthetic care unit. This was a single centre randomised trial of 200 patients at risk of postoperative pulmonary complications (PPCs) which showed that, while sugammadex was associated with a significant reduction in postoperative paralysis, this did not lead to a reduction in PPCs (10). This study did however show a reduction in 30-day readmission rates with sugammadex use.

In addition to this single randomised trial, there have been three recent large trials utilising large data sets, also producing conflicting results. The post-anaesthesia pulmonary complications after use of muscle relaxants (POPULAR) study was a prospective observational study in 22,803 patients receiving general anaesthesia. Their results showed that while the use of neuromuscular blocking agents was associated with an increase in PPCs (11), neither the use of sugammadex, nor extubation without measurable residual paralysis, was associated with a reduction in PPCs. The sugammadex versus neostigmine for reversal of neuromuscular blockade and postoperative pulmonary complications (STRONGER) trial was a multicentre observational matched-cohort study with 22,586 patients in each of the sugammadex and neostigmine groups. This showed that the use of sugammadex was associated with a reduction in PPCs and a reduction in the risk of pneumonia and respiratory failure (12). Finally, a retrospective historical before and after cohort study in 10,491 patients showed no difference in the rates of PPCs within a single institution after swapping from neostigmine to sugammadex based reversal (13).

These conflicting results provide equipoise as to the clinical effect of sugammadex on postoperative pulmonary complications in modern anaesthetic practice, and justify the need for a randomised controlled trial to determine whether the use of sugammadex improves patient outcomes. 
P-PERSoN was designed as a prospective multi-centre, double-blinded, randomised controlled trial to evaluate if sugammadex use reduced the incidence of postoperative pulmonary complications compared to neostigmine reversal.

\section{Methods}

\section{Trial Design}

Across two centres (both a large quaternary public hospital and private hospital setting), adults admitted for surgical operations requiring muscle paralysis were enrolled in a prospective, double-blind, randomised controlled trial comparing specific outcomes following reversal with sugammadex or neostigmine. The study was approved by the Northern Sydney Local Health District Human Research Ethics Committee (RESP/16/289) and the North Shore Private Hospital Ethics Committee (2017-001).

The trial was registered at the Australian New Zealand Clinical Trials Registry (www.anzctr.org.au) \# ACTRN12616000063415 (21/01/2016) and The U.S. National Library of Medicine (www.ClinicalTrials.gov) \#NCT02825576 (07/07/2016). The trial was compliant with the Australian National Health and Medical Research Council (NHMRC) statement on ethical conduct in human research (2007) (14) and the note for guidance on good clinical practice (CPMP/ICH-135/95) (15).

\section{Participants}

Screening for eligible patients was conducted by examining operating theatre and preadmission clinic schedules for each day. Inclusion criteria were: patients over 18 years of age, planned to undergo noncardiac surgery with an expected operative time of $>2$ hours, use of endotracheal intubation, and an expected hospital length of stay greater than one night. These criteria were designed to preferentially select patients who would likely be at intermediate or high risk for PPCs (16).

Exclusion criteria were: patients previously recruited, patient weight $>200 \mathrm{~kg}$, planned expectation to remain intubated postoperatively, and those with known hypersensitivity reactions or contraindications to any of the study drugs.

Patient recruitment occurred during a preoperative consultation, either in a preadmission clinic or on a hospital ward, prior to their transfer to the operating theatre.

\section{Interventions}

Patients were randomly assigned into one of two groups; the sugammadex group or the neostigmine group. At the end of surgery, the participant received a blinded reversal dose via a $10 \mathrm{ml}$ syringe with administration of $1 \mathrm{~mL} / 10 \mathrm{~kg}$ of the blinded solution. The solution was standardised to contain the protocol's reversal dose that would equate to either $2 \mathrm{mg} / \mathrm{kg}$ of sugammadex or $50 \mathrm{mcg} / \mathrm{kg}$ neostigmine with $10 \mathrm{mcg} / \mathrm{kg}$ glycopyrrolate. 
The study protocol required the use of intraoperative neuromuscular twitch (NMT) monitoring to ensure return of a train-of-four count (TOFC) greater than or equal to 2 prior to reversal, and restricted the use of neuromuscular blockers to rocuronium or vecuronium, at the choice of the individual anaesthetist.

There were no restrictions on the mode of anaesthesia, analgesia, use of postoperative nausea and vomiting (PONV) prophylaxis, or timing of neuromuscular blockade reversal.

\section{Randomisation}

Patients were allocated to treatment group via a computer-based simple randomisation software (randomizer.org). Group allocations were placed in a sealed opaque envelope by a research team member who was not involved in patient treatment or follow up. Prior to reversal, the treating anaesthetist recruited a member of the hospital clinical staff to open the next available randomisation envelope, draw up the allocated reversal agent based on the enclosed instructions, and deliver this to the treating anaesthetist. This individual was then not involved in further management of the trial patient.

\section{Blinding}

The patient, treating anaesthetist, all care providers, and research personnel assessing patient outcomes were all blinded to the patient's treatment allocation.

\section{Outcomes}

The primary outcome was the incidence of PPCs (as defined by the European Perioperative Clinical Outcome (EPCO) statement (17) in the first three days postoperatively (Table 1).

Secondary outcomes were; patient reported quality of recovery at day 1 and 30 utilising the QoR-15 (18), the incidence of PONV, and the incidence of a defined set of airway events in the post anaesthetic care unit (PACU). In addition to the Quality-of-Recovery-15 score (QoR-15) at day 30, patients were also asked if they had also received either antibiotics or bronchodilators to treat a chest infection (or if previously on bronchodilators, whether they had increased their usage or dose) in the interim. The hospital discharge summary was reviewed to identify the diagnosis of a respiratory infection or associated complications and to calculate hospital length of stay.

Subgroup analysis was constructed to facilitate stratification into groups based on the risk of PPC, as defined by the Assess Respiratory Risk in Surgical Patients in Catalonia (ARISCAT) score (16), the risk of PONV as defined by the Apfel score (19), the use of Total Intravenous Anaesthesia (TIVA) vs volatile based anaesthesia, patient Body Mass Index (BMI), and American Society of Anesthesiologists (ASA) Class.

Table 1 outlines the EPCO definition, ARISCAT and Apfel scores, PACU Airway Events and the PONV scoring systems used.

Table 1 - Definitions used for outcome measurements and risk stratification 


\section{European Perioperative Clinical Outcome (EPCO) definitions of Post-operative Pulmonary. Complications (17).}
Respiratory
Patient has received antibiotics for suspected respiratory infection and met one infection or more of the following criteria: new or changed sputum, new or changed lung opacities, fever, white blood cell count $>12 \times 10^{9} /$ litre
Respiratory failure arterial oxyhaemoglobin saturation measured with pulse oximetry $<90 \%$ and needing oxygen therapy Postoperative $\mathrm{PaO}_{2}<60 \mathrm{~mm} \mathrm{Hg}$ on room air, $\mathrm{PaO}_{2}: \mathrm{FiO}_{2}$ ratio $<300 \mathrm{~mm} \mathrm{Hg}$ ), or Pleural
effusion Chest radiograph showing blunting of costophrenic angle, loss of sharp silhouette of ipsilateral hemidiaphragm in upright position, evidence of
displacement of adjacent anatomical structures, or (in supine position) hazy
opacity in one hemithorax with preserved vascular shadows
Atelectasis Lung opacification with shift of mediastinum, hilum, or hemidiaphragm towards affected area, and compensatory over-inflation in adjacent non-atelectatic lung
Pneumothorax

$$
\text { Air in pleural space with no vascular bed surrounding visceral pleura }
$$

Assess Respiratory Risk in Surgical Patients in Catalonia (ARISCAT) Score for risk prediction of postoperative pulmonary complications (16).

\begin{tabular}{ll} 
Risk Factor & Score \\
\hline Age $51-80$ & 3 \\
\hline Age $>80$ & 16 \\
\hline Preoperative Sp02 91-95\% & 8 \\
\hline Preoperative Sp02 $\leq 90 \%$ & 24 \\
\hline Respiratory infection in past 1 month & 17 \\
\hline Preoperative Haemoglobin<10 gm/dl & 11 \\
\hline Upper abdominal incision & 15 \\
\hline Intrathoracic incision & 24 \\
\hline Surgery Duration 2-3 hours & 16 \\
\hline Surgery Duration $>3$ hours & 23 \\
\hline Emergency Procedure & 8
\end{tabular}

Total Score - Low risk: $<26$, Intermediate risk: 26-44, High risk: $\geq 45$

Apfel Score for risk prediction of PONV (19). 
Risk Factors (1 point each) - Female sex, History of PONV or motion sickness, non-smoker, postoperative opioid treatment planned.

Total score and risk stratification

\begin{tabular}{cl}
0 & Minimal Risk \\
\hline 1 & Low Risk \\
\hline 2 & Intermediate Risk \\
\hline 3 & High Risk \\
\hline 4 & Very High Risk
\end{tabular}

Definitions of postoperative care unit events

PACU Events

- Any desaturation to $\mathrm{SpO} 2<90 \%$

- Need for manual airway support

- Need for oropharyngeal or nasopharyngeal airway

- Need for reintubation in PACU

- Need for anaesthetist to review the patient

- Unplanned ICU admission

\section{PONV score}

1. no PONV

2. PONV responsive to antiemetics

3. PONV unresponsive to antiemetics

$\mathrm{PaO} 2$-partial pressure of arterial oxygen, FiO2 - Fraction of inspired oxygen, SpO2 - peripheral capillary oxygen saturation, PONV - Postoperative Nausea and Vomiting, PACU - Post Anaesthesia Care Unit, ICU - Intensive Care Unit

\section{Sample Size}

Prior to the design of this trial, the largest published prospective study of PPCs showed an overall incidence of $7.9 \%$, stratified into low risk (3.4\%), intermediate risk (13.0\%), and high risk (38.0\%) (16).

Given this study was designed to include intermediate and high-risk patients, a conservative estimate of a $7 \%$ baseline incidence of PPCs was used. Based on prior retrospective work, showing an odds ratio of 0.28 (20), sugammadex would be expected to reduce the incidence of PPCs from 7-1.96\%. Choosing a conservative reduction to an incidence of $3.5 \%$, produced a clinically relevant Number Needed to Treat 
(NNT) of 29. Accepting an alpha error of 0.05 , a beta error of 0.2 , and estimating $5 \%$ incomplete data and loss to follow up resulted in a target sample size of 976 patients (21).

\section{Statistical analysis}

The presence of a PPCs, and the presence of PACU events were assessed as categorical variables, QoR15 score and hospital stay were assessed as continuous variables, and PONV score was assessed as an ordinal variable. Continuous variables were assessed with two tailed t-tests, categorical and ordinal data was assessed by Chi-squared analysis of contingency tables (or Fisher's exact test when $n<5$ in any outcome).

Binomial regression analysis was planned for categorical outcomes in subgroup analyses, with logistic regression for the ordinal variables.

Interim analysis on the primary outcome was planned when 488 ( $50 \%$ of patients) had been randomised and had completed the 30 day follow-up. The Haybittle-Peto approach was planned to be used, where the trial will be ended with symmetric stopping boundaries at $\mathrm{P}<0.001$. The trial was overseen by a Data and Safety Monitoring Committee (DSMC) who would advise on early trial cessation in case of futility of treatment effect or safety concerns.

\section{Results}

\section{Participant Flow}

The trial was commenced in December 2018 and was subsequently paused in March 2019 due to the abrupt resignation of research personnel needed to support the trial. This was followed by a prolonged approval and recruitment process to replace them. The recruitment process was completed in January 2020, and while planning to restart, the COVID-19 epidemic occurred. Due to the delay in restarting and absence of funding, the trial investigators made the decision to terminate the trial early and the results analysed to inform future large-scale randomised controlled trials (RCTs) comparing similar outcomes with the use of sugammadex or neostigmine.

A total of 51 patients were screened for eligibility, 33 ( $65 \%$ of screened patients) met criteria and were enrolled. There were three patients who had their surgery following study termination, leaving 30 patients achieving randomisation. Study drug was successfully delivered to $100 \%$ of randomised patients. All patients had primary outcome data collected, with a $97 \%$ data completion rate for in-hospital data, and a 93\% completion rate for 30-day data collection (Figure 1).

Baseline demographics and clinical characteristics were similar in both groups (Table 2). 
Table 2

- Demographic characteristics of study participants

\begin{tabular}{|c|c|c|}
\hline & Sugammadex & Neostigmine \\
\hline Age (years) mean (SD) & $57+/-7$ & $58+/-7$ \\
\hline Female Sex (n/total) & $68 \%(13 / 19)$ & $45 \%(5 / 11)$ \\
\hline \multicolumn{3}{|l|}{ ASA Class (n/total) } \\
\hline 1 & $11 \%(2 / 19)$ & $9 \%(1 / 11)$ \\
\hline 2 & $68 \%(13 / 19)$ & $55 \%(6 / 11)$ \\
\hline 3 & $21 \%(4 / 19)$ & $36 \%(4 / 11)$ \\
\hline 4 & $0 \%(0 / 19)$ & $0 \%(0 / 11)$ \\
\hline Height (cm) & $165+/-4$ & $171+/-6$ \\
\hline Weight (kg) & $78+/-8$ & $81+/-18$ \\
\hline BMI & $28+/-2$ & $28+/-5$ \\
\hline Current Smoker (n/total) & $5 \%(1 / 19)$ & $9 \%(1 / 11)$ \\
\hline Duration of Surgery (min) & $197+/-25$ & $204+/-51$ \\
\hline \multicolumn{3}{|l|}{ Relaxant Used (n/total) } \\
\hline Rocuronium & $21 \%(4 / 19)$ & $45 \%(5 / 11)$ \\
\hline Vecuronium & $79 \%(15 / 19)$ & $55 \%(6 / 11)$ \\
\hline \multicolumn{3}{|l|}{ PONV Prophylaxis (n/total) } \\
\hline None & $0 \%(0 / 19)$ & $18 \%(2 / 11)$ \\
\hline Dexamethasone & $26 \%(5 / 19)$ & $18 \%(2 / 11)$ \\
\hline Ondansetron & $11 \%(2 / 19)$ & $27 \%(3 / 11)$ \\
\hline Dexamethasone/Ondansetron & $58 \%(11 / 19)$ & $36 \%(4 / 11)$ \\
\hline Dexamethasone/Droperidol & $5 \%(1 / 19)$ & $0 / 11(0 / 11)$ \\
\hline \multicolumn{3}{|l|}{ ARISCAT Risk Score (n/total) } \\
\hline Low & $33 \%(6 / 18)^{\star}$ & $27 \%(3 / 11)$ \\
\hline Intermediate & $67 \%(12 / 18)^{\star}$ & $45 \%(5 / 11)$ \\
\hline
\end{tabular}

*One patient had no preoperative $\mathrm{Hb}$, therefore could not calculate ARISCAT score. SD - standard deviation, ASA - American Society of Anesthesiologists, ARISCAT - Assess Respiratory Risk in Surgical Patients in Catalonia, BMI - Body-Mass Index, PONV - Postoperative nausea and vomiting. 


\begin{tabular}{|c|c|c|}
\hline & Sugammadex & Neostigmine \\
\hline High & $0 \%(0 / 18)^{\star}$ & $27 \%(3 / 11)$ \\
\hline \multicolumn{3}{|c|}{ Apfel Risk Score (n/total) } \\
\hline Minimal & $0 \%(0 / 19)$ & $0 \%(0 / 11)$ \\
\hline Low & $16 \%(3 / 19)$ & $36 \%(4 / 11)$ \\
\hline Intermediate & $74 \%(14 / 19)$ & $64 \%(7 / 11)$ \\
\hline High & $11 \%(2 / 19)$ & $0 \%(0 / 11)$ \\
\hline Very High & $0 \%(0 / 19)$ & $0 \%(0 / 11)$ \\
\hline \multicolumn{3}{|c|}{$\begin{array}{l}\text { *One patient had no preoperative Hb, therefore could not calculate ARISCAT score. SD - standard } \\
\text { deviation, ASA - American Society of Anesthesiologists, ARISCAT - Assess Respiratory Risk in Surgical } \\
\text { Patients in Catalonia, BMI - Body-Mass Index, PONV - Postoperative nausea and vomiting. }\end{array}$} \\
\hline
\end{tabular}

All randomised patients received the study drug as per their assigned group. There was one violation in the sugammadex group, where a patient received the assigned reversal agent despite having only one twitch on NMT monitoring (reported as being suitable for extubation on clinical grounds by the treating anaesthetist). One patient had no PACU data, as they unexpectedly remained intubated and subsequently were transferred directly to the Intensive Care Unit (ICU) (neostigmine group). Two patients in the sugammadex group were lost to follow up for 30-day data collection.

Due to the early termination and low numbers, there were no interim analyses performed and subgroup analysis was not performed. While the number of participants recruited for participation do not meet those needed to power the study's analysis, we present their results below.

\section{Primary Outcome - Postoperative Pulmonary Complications}

One patient in the neostigmine group, and no patients in the sugammadex group, had a documented PPC (9\% [95\% Cl 0-41\%] vs 0\% [0-17\%]). RR $5.0(95 \% \mathrm{Cl} 0.22-113) \mathrm{p}=0.37$.

\section{Quality of Recovery}

The neostigmine group reported a mean QoR-15 score (95\% Cl) of 87 (60-104) at day 1, and 133 (120$146)$ at day 30 , the mean $(95 \% \mathrm{Cl})$ QoR score in the sugammadex group was $(102(88-116) p=0.21$ and $129(117-142) \mathrm{p}=0.61)$ respectively.

\section{Postoperative Nausea and Vomiting}

Postoperative nausea and vomiting was reported in one subject $(1 / 10)$ in the neostigmine group vs three patients in the sugammadex group (3/19) RR $1.6(95 \% \mathrm{Cl} 0.18-13.3) \mathrm{p}=0.99$

\section{Complications in the Postoperative Anaesthesia Care Unit}


There was one incidence of desaturation to $<90 \%$ in both the sugammadex and neostigmine groups $(5.3 \%$ vs $10 \% p=0.99)$. There were no other airway complications detected in either group. Two patients in each of the groups required a review by an anaesthetist (sugammadex group $10.6 \%$ vs neostigmine group $20 \% p=0.59$ ). In the sugammadex group, one review was for bradycardia, while a second was for shortness of breath. Subsequent reviews for participants in the neostigmine group were for a new onset wheeze with desaturation in one patient, and for uncontrolled pain in a second patient.

\section{Hospital Stay}

Hospital length of stay was similar between both groups. (Group S: 4.2 (2.8-5.6) days, Group N: 5.6 (2.29.0) days, $p=0.44$ ).

\section{Postoperative Follow Up}

One patient was found to have received antibiotics for a chest infection at day 30 in the sugammadex group, with no patients in the neostigmine group reporting the need for antibiotics and no patients in either group reporting new or increased bronchodilator therapy in the 30 days postoperatively.

Table 3 provides a summary of all outcome data collected.

Table 3

- Outcome Data

\begin{tabular}{|c|c|c|c|}
\hline & Sugammadex & Neostigmine & $\begin{array}{l}\text { p- } \\
\text { value }\end{array}$ \\
\hline Postoperative Pulmonary Complication & $0(0-17) \%$ & $9(0-41) \%$ & 0.37 \\
\hline PONV Score $>1$ & $16(3-40) \%$ & $10(0-44) \%$ & 0.99 \\
\hline Events in PACU (\%) & $16(3-40) \%$ & $27(6-60) \%$ & 0.59 \\
\hline QOR-15 Day 1 & $102(88-116)$ & $87(60-104)$ & 0.21 \\
\hline QoR-15 Day-30 & $\begin{array}{l}129(117- \\
142)\end{array}$ & $\begin{array}{l}133(120- \\
146)\end{array}$ & 0.61 \\
\hline Need for postoperative antibiotics to day 30 & $6(0-29) \%$ & $0(0-28) \%$ & 0.99 \\
\hline Need for new or increased bronchodilators to day 30 & $0(0-19) \%$ & $0(0-28) \%$ & 0.99 \\
\hline $\begin{array}{l}\text { Hospital Discharge Summary diagnosis of respiratory } \\
\text { infection }\end{array}$ & $0(0-18) \%$ & $0(0-28) \%$ & 0.99 \\
\hline Hospital Length of Stay (days) & $4.2(2.8-5.6)$ & $5.6(2.2-9.0)$ & 0.44 \\
\hline
\end{tabular}

\section{Discussion}


This randomised controlled trial was constructed to assess neuromuscular blockade reversal with sugammadex compared to neostigmine in patients who were at intermediate to high risk of PPC. The trial was ceased early due to resource constraints and COVID-19. In the small numbers that were recruited there was no difference detected in the rates of postoperative pulmonary complications following reversal with sugammadex when compared with reversal with neostigmine. There was also no difference found between groups in terms of patient quality of recovery, as measured by the QoR-15 score, postoperative nausea and vomiting, a composite of PACU events and hospital length of stay and the need for chest infection treatment in the first 30 days postoperatively. Given the small sample size before trial termination, there was not an ability to assess for clinically meaningful or statistically significant differences in outcomes, and as such, the outcomes of this study cannot be directly translated into clinical care.

Despite these limitations, the experience of designing and initiating this trial has led to a number of practical recommendations for future attempts at similar endeavours. Firstly, a more clinically relevant patient-centred primary outcome would be recommended. Days Alive and at Home to Day $30\left(\mathrm{DAH}_{30}\right)$, is a validated, patient-centred outcome that is associated with surgical complexity, in-hospital complications, and longer-term outcomes, which can also be easily linked to economic outcomes (22). Additionally, good published data exists in a variety of surgical populations of mean and variability for $\mathrm{DAH}_{30}$ to provide for power analysis (23). Future studies should also consider assessing a return to normal function, including normal levels of work/study, to further assess any social and economic implications of clinical outcomes/impacts.

Finally, using the QOR-15 at 30 days as a measure of quality of recovery needs an adaptation for out of hospital use, as one of the included questions is worded to enquire about "support from hospital doctors and nurses", which is not relevant after discharge. A modified QoR-15 questionnaire to assess "receiving support from healthcare services, if you needed it" could address this incompatibility, or the use of an alternative validated quality of life metric.

We have reported the study according to randomised study standards as recommended by the Consolidated Standards of Reporting Trials (CONSORT) group (24).

\section{Conclusions}

We were not able to discern whether sugammadex compared to neostigmine was associated with a reduced rate of post-operative pulmonary complications. Future research to definitively address this question is needed and appears feasible with a well-designed and adequately funded study.

\section{Abbreviations}

ARISCAT Assess Respiratory Risk in Surgical Patients in Catalonia

ASA American Society of Anesthesiologists

Page 12/18 
BMI Body Mass Index

CONSORT Consolidated Standards of Reporting Trials

COVID - coronavirus disease

DAH $_{30}$ Days Alive and at Home to Day 30

DSMC Data and Safety Monitoring Committee

EPCO European Perioperative Clinical Outcome

ICU Intensive Care Unit

NHMRC National Health and Medical Research Council

NMT neuromuscular twitch

NNT Number Needed to Treat

PACU post anaesthetic care unit

PONV postoperative nausea and vomiting

POPULAR post-anaesthesia pulmonary complications after use of muscle relaxants

PPC postoperative pulmonary complication

QoR-15 Quality-of-Recovery-15 score

RCT randomised controlled trial

STRONGER sugammadex versus neostigmine for reversal of neuromuscular blockade and postoperative pulmonary complications

TIVA Total Intravenous Anaesthesia

TOFC train-of-four count

\section{Declarations}

\section{Ethics approval and consent to participate}

The study was approved by the Northern Sydney Local Health District Human Research Ethics Committee (RESP/16/289) and the North Shore Private Hospital Ethics Committee (2017-001). Informed consent was obtained from all participants. 
Consent for publication

Not Applicable

Availability of data and materials

The datasets used and/or analysed during the current study are available from the corresponding author on reasonable request.

Competing interests

The authors declare that they have no competing interests

\section{Funding}

There was no specific funding for this study

\section{Authors' contributions}

BO made substantial contributions to the conception and design of the work; the acquisition, analysis and interpretation of data and drafted the work or substantively revised it.

MD made substantial contributions to the design of the work; the acquisition, analysis and interpretation of data and drafted the work or substantively revised it.

CF made substantial contributions to the conception and design of the work and drafted the work or substantively revised it

GK made substantial contributions to the conception and design of the work and drafted the work or substantively revised it

AD made substantial contributions to the conception and design of the work and drafted the work or substantively revised it

All authors read and approved the final manuscript

\section{Acknowledgements}

We would like to thank the following team members for their contributions to this trial.

Participating Centres: Royal North Shore Hospital, North Shore Private Hospital.

Research Coordinator: Ms Courtney Player. Data Safety and Management Committee: Professor Peter Kam and Dr Ross Wallace

\section{References}


1. Bissinger U, Schimek F, Lenz G. Postoperative residual paralysis and respiratory status: a comparative study of pancuronium and vecuronium. Physiol Res. 2000;49(4):455-62.

2. Murphy GS, Szokol JW, Marymont JH, Greenberg SB, Avram MJ, Vender JS. Residual neuromuscular blockade and critical respiratory events in the postanesthesia care unit. Anesth Analg. 2008;107(1):130-7.

3. Murphy GS, Szokol JW, Franklin M, Marymont JH, Avram MJ, Vender JS. Postanesthesia care unit recovery times and neuromuscular blocking drugs: a prospective study of orthopedic surgical patients randomized to receive pancuronium or rocuronium. Anesth Analg. 2004;98(1):193-200. table of contents.

4. Berg H, Roed J, Viby-Mogensen J, Mortensen CR, Engbaek J, Skovgaard LT, et al. Residual neuromuscular block is a risk factor for postoperative pulmonary complications. A prospective, randomised, and blinded study of postoperative pulmonary complications after atracurium, vecuronium and pancuronium. Acta Anaesthesiol Scand. 1997;41(9):1095-103.

5. Cammu GV, Smet V, De Jongh K, Vandeput D. A prospective, observational study comparing postoperative residual curarisation and early adverse respiratory events in patients reversed with neostigmine or sugammadex or after apparent spontaneous recovery. Anaesth Intensive Care. 2012;40(6):999-1006.

6. Booij LH. Cyclodextrins and the emergence of sugammadex. Anaesthesia. 2009;64(Suppl 1):31-7.

7. Ledowski T, Hillyard S, O'Dea B, Archer R, Vilas-Boas F, Kyle B. Introduction of sugammadex as standard reversal agent: Impact on the incidence of residual neuromuscular blockade and postoperative patient outcome. Indian J Anaesth. 2013;57(1):46-51.

8. Brueckmann B, Sasaki N, Grobara P, Li MK, Woo T, de Bie J, et al. Effects of sugammadex on incidence of postoperative residual neuromuscular blockade: a randomized, controlled study. $\mathrm{Br} \mathrm{J}$ Anaesth. 2015;115(5):743-51.

9. Kotake Y, Ochiai R, Suzuki T, Ogawa S, Takagi S, Ozaki M, et al. Reversal with sugammadex in the absence of monitoring did not preclude residual neuromuscular block. Anesth Analg. 2013;117(2):345-51.

10. Togioka BM, Yanez D, Aziz MF, Higgins JR, Tekkali P, Treggiari MM. Randomised controlled trial of sugammadex or neostigmine for reversal of neuromuscular block on the incidence of pulmonary complications in older adults undergoing prolonged surgery. Br J Anaesth. 2020;124(5):553-61.

11. Kirmeier E, Eriksson LI, Lewald H, Jonsson Fagerlund M, Hoeft A, Hollmann M, et al. Post-anaesthesia pulmonary complications after use of muscle relaxants (POPULAR): a multicentre, prospective observational study. Lancet Respir Med. 2019;7(2):129-40.

12. Kheterpal S, Vaughn MT, Dubovoy TZ, Shah NJ, Bash LD, Colquhoun DA, et al. Sugammadex versus Neostigmine for Reversal of Neuromuscular Blockade and Postoperative Pulmonary Complications (STRONGER): A Multicenter Matched Cohort Analysis. Anesthesiology. 2020;132(6):1371-81.

13. Li G, Freundlich RE, Gupta RK, Hayhurst CJ, Le CH, Martin BJ, et al. Postoperative Pulmonary Complications' Association with Sugammadex versus Neostigmine: A Retrospective Registry 
Analysis. Anesthesiology. 2021;134(6):862-73.

14. The National Health and Medical Research Council tARCaUA. National Statement on Ethical Conduct in Human Research. Canberra: Commonweath of Australia; 2007. (Updated 2018).

15. (ICH) ICfHoTRfPfHU. Integrated Addendun to ICH E6(R1): Guideline for Good Clinical Practice. https://ichgcp.net/2016.

16. Mazo V, Sabate S, Canet J, Gallart L, de Abreu MG, Belda J, et al. Prospective external validation of a predictive score for postoperative pulmonary complications. Anesthesiology. 2014;121(2):219-31.

17. Jammer I, Wickboldt N, Sander M, Smith A, Schultz MJ, Pelosi P, et al. Standards for definitions and use of outcome measures for clinical effectiveness research in perioperative medicine: European Perioperative Clinical Outcome (EPCO) definitions: a statement from the ESA-ESICM joint taskforce on perioperative outcome measures. Eur J Anaesthesiol. 2015;32(2):88-105.

18. Stark PA, Myles PS, Burke JA. Development and psychometric evaluation of a postoperative quality of recovery score: the QoR-15. Anesthesiology. 2013;118(6):1332-40.

19. Apfel CC, Laara E, Koivuranta M, Greim CA, Roewer N. A simplified risk score for predicting postoperative nausea and vomiting: conclusions from cross-validations between two centers. Anesthesiology. 1999;91(3):693-700.

20. Olesnicky BL, Traill C, Marroquin-Harris FB. The effect of routine availability of sugammadex on postoperative respiratory complications: a historical cohort study. Minerva Anestesiol. 2017;83(3):248-54.

21. Faul F, Erdfelder E, Lang AG, Buchner A. G*Power 3: a flexible statistical power analysis program for the social, behavioral, and biomedical sciences. Behav Res Methods. 2007;39(2):175-91.

22. Jerath A, Austin PC, Wijeysundera DN. Days Alive and Out of Hospital: Validation of a Patientcentered Outcome for Perioperative Medicine. Anesthesiology. 2019;131(1):84-93.

23. Myles PS, Shulman MA, Heritier S, Wallace S, Mcllroy DR, McCluskey S, et al. Validation of days at home as an outcome measure after surgery: a prospective cohort study in Australia. BMJ Open. 2017;7(8):e015828.

24. Schulz KF, Altman DG, Moher D, Group C. CONSORT 2010 statement: updated guidelines for reporting parallel group randomised trials. BMJ. 2010;340:c332.

\section{Figures}




\section{O N S O R T}

TRANSPARENT REPORTING Of TRIALS

\section{CONSORT 2010 Flow Diagram}

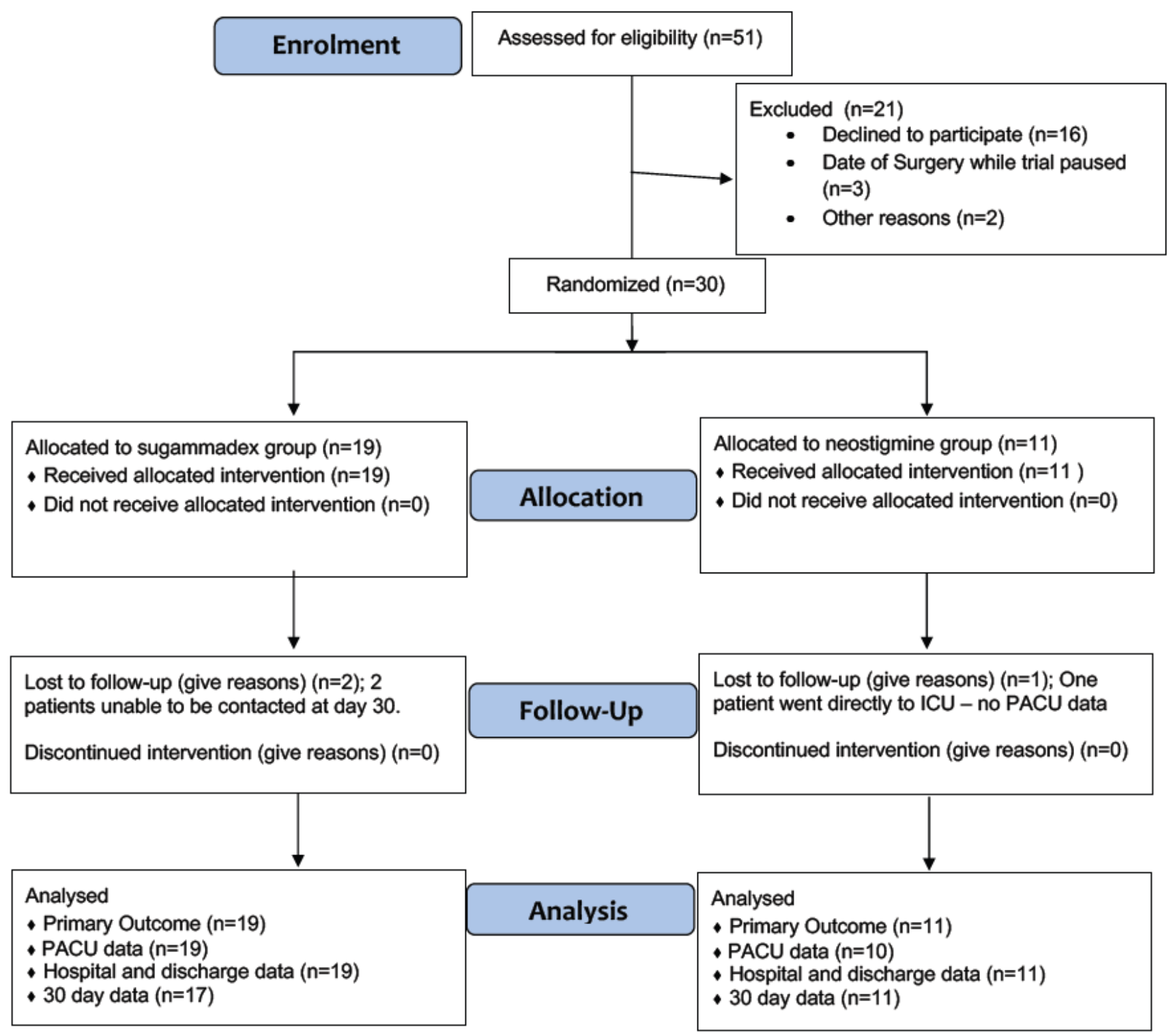

\section{Figure 1}

CONSORT Flowchart for the P-PERSoN trial PACU - Post Anaesthesia Care Unit, ICU - Intensive Care Unit

\section{Supplementary Files}

This is a list of supplementary files associated with this preprint. Click to download. 
- CONSORT2010ChecklistPPERSoN.doc

- Protocol2.1.pdf 